\title{
A Liquid Crystal Multifilter System for Diascopic Microscopy
}

\author{
Douglas Clark* and Brian Brown
}

Paedia LLC, 327 Lake Street, San Francisco, CA 94118

^dclark@paedia.com

\begin{abstract}
An electrically addressable, transmissive liquid crystal display device is placed in the diascopic illumination path of a microscope. The display emulates the disks, orifices, and color filters used in well-known contrast-enhancing darkfield, brightfield, Rheinberg, and oblique illuminations. Each illumination modality is selectable, sizable, and locatable by software in a computing device that is connected to the display. Tedious construction and placement of pre-cut disks, orifices, and filters are no longer needed. Light leaving the liquid crystal display is polarized so with the addition of an analyzer a subject may be viewed in polarized light.
\end{abstract}

\section{Introduction}

Various subjects such as protozoa, plant and animal cells, and the like are nearly transparent when viewed with ordinary brightfield diascopic illumination. Light is only weakly absorbed in these nearly transparent subjects so that they are not differentiated from the background light that illuminates them. In the past, various image contrast-enhancing techniques have been used to render these subjects visible and more easily studied. Dyes such as hematoxylin and eosin have been used to respectively stain the nuclei and cytoplasm of cells for improved visualization. A number of illumination- and light-processing techniques are also used to improve visualization with or without staining of subjects. These techniques include darkfield, Rheinberg, oblique, monochrome, polarized, phase-contrast, differential-interference-contrast, and intensity-modulationcontrast illumination. Each of these techniques requires its own add-on microscope accessory, and many accessories must be adjusted to match the numerical aperture of the objective lens being used. Because of these encumbrances, microscopists often pass up an opportunity to quickly view a subject in a variety of perspectives.

This article describes the Diabloc ${ }^{\mathrm{TM}}$ Optic Multifilter (DOM), a digital illumination filter for diascopic microscopy. It switches between brightfield, darkfield, Rheinberg, oblique, and monochrome illuminations by the simple rotation of a knob. Adjustments for different colors and different numerical apertures of objective lenses in darkfield and Rheinberg illuminations are also done by rotating a knob. With our apparatus, a microscopist can quickly move among these contrastenhancing methods and see what they would otherwise miss. The following paragraphs describe the physical setup for the diascopic contrast-enhancing methods encompassed in the DOM.

\section{Types of Illumination}

Brightfield illumination. Brightfield illumination is the simplest technique used in microscopy. A subject is illuminated from one side, and light transmitted through the subject is viewed from the opposite side.
Although this technique is widely used, it has limitations in the viewing of transparent or very translucent subjects because there is little contrast between the subject and the background light.

Darkfield illumination. Darkfield illumination enhances contrast by obliquely illuminating a subject. A central light block is inserted between a microscope's light source and condenser so that only light that is reflected or refracted in a subject reaches the microscope's objective lens. There is no direct light path from the microscope's light source, through or around a subject, so the subject is revealed in a bright image on a dark background.

In the past, numerous devices have been used for darkfield illumination. These range from a simple "spider light stop," an opaque disk that is positioned at the light entrance of a condenser, to specialized devices such as a cardioid darkfield condenser [1]. The latter has a number of internal components and is more expensive than the simple opaque disc light stop.

In order to optimize a darkfield opaque disk setup, the diameter of the disk must be tailored to the numeric aperture of the microscope's objective lens. If the disk is too small, stray light will enter the microscope's objective lens and reduce the contrast of the resultant image. If the disk is too large, the amount of light available for viewing an image is less than optimum. In the past, disks of varying sizes were used. When an objective lens was changed, it was necessary to change disks [1].

Rheinberg illumination. Rheinberg illumination is an extension of darkfield illumination. An optical filter material of a first color replaces the central light block of a darkfield opaque disk, and another optical filter of a second color covers the outer light path that obliquely illuminates a subject [2]. The filters are located in the same position as a darkfield light block. In this technique, the first color of the central light path forms the background of an image, while the second color of the outer light path illuminates the subject. In the past, the filters were laboriously cut from gelatin color filter materials. As with conventional darkfield, for optimum results the diameter of the central filter must be tailored to the numerical aperture of the objective lens.
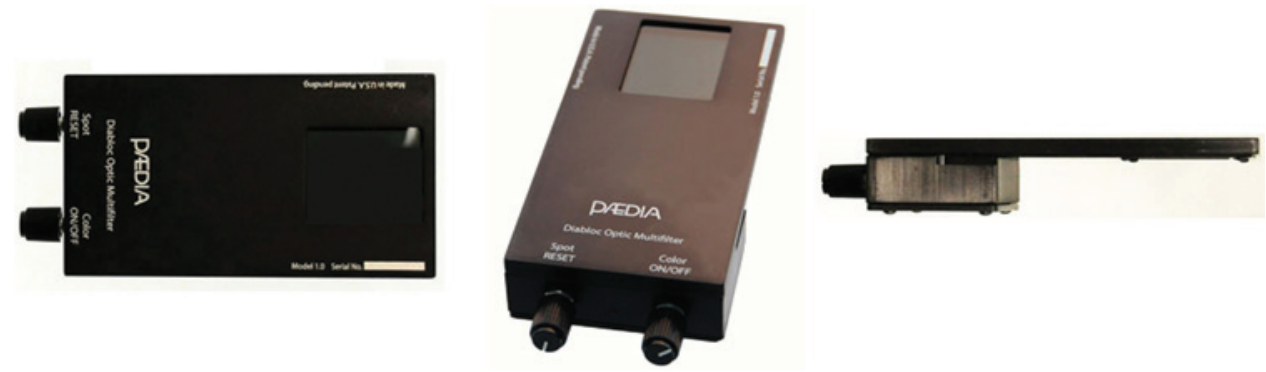

Figure 1: Top, perspective, and side views of the DOM. The Multifilter is $15 \times 7.5 \times 2.8 \mathrm{~cm}$ in length, width, and height. 


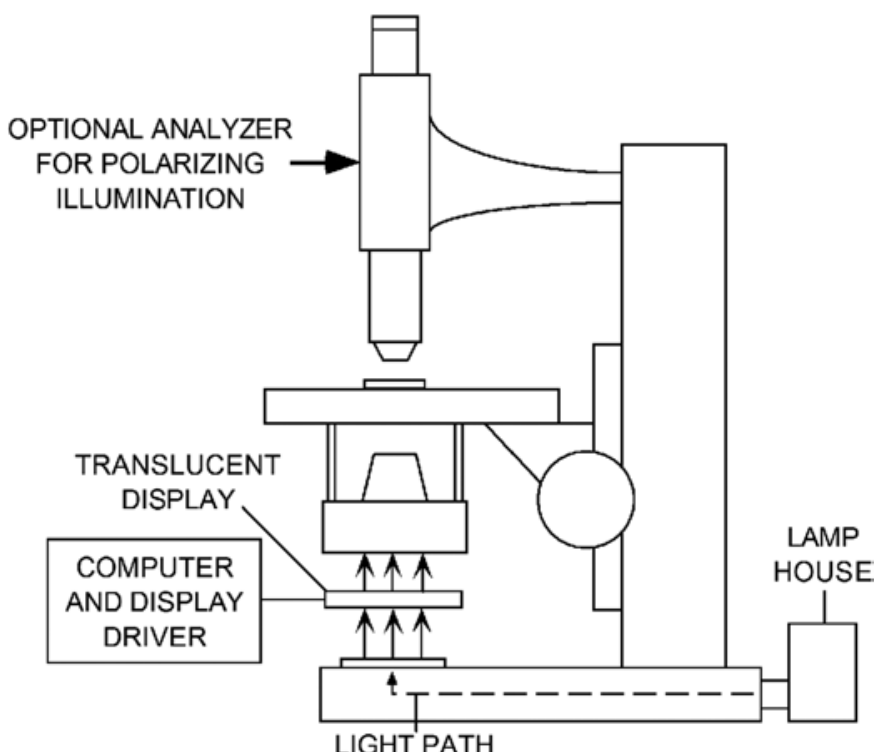

Figure 2: Placement of the DOM between the base and the condenser in the light path of a microscope. The LCD of the Multifilter replaces the opaque or colored disks used in darkfield, Rheinberg, and oblique illumination.

Oblique illumination. In oblique illumination, light that illuminates a subject is selected from an off-center portion of the collimated beam from the microscope's light source. In one method, the lamp that illuminates the subject is moved off-center so that the subject is illuminated from only one side. In another method, the lamp is centered, and an off-center slit passes light into the microscope's condenser. In both cases, details of the subject are rendered in pseudo-relief for a 3D-like appearance [2].

Monochrome illumination. In monochrome illumination, a colored filter is interposed between a microscope's light source and its condenser. Contrast is enhanced when a filter preferentially blocks a portion of the visible light spectrum that illuminates a subject. For example, a didymium filter [3] is used in viewing tissue subjects that have been stained with hematoxylin and eosin. In general, hematoxylin stains cell nuclei blue, and eosin stains cytoplasm and the extracellular matrix a yellow-magenta color. The didymium filter blocks light with wavelengths near the yellow portion of the spectrum while passing light in the red and blue portions. The result is greater contrast between the cell nuclei and the surrounding material.

Polarized illumination. In polarized illumination, light shining through a first polarizing filter illuminates a subject, and light leaving the illuminated subject is viewed through a second polarizing filter, also called an analyzer. In the absence of a subject, when the polarizer and analyzer axes are crossed, no light leaves the analyzer, so the view through a microscope is completely dark. However, when a birefringent subject is placed between the polarizer and analyzer, an image is seen with colors and intensities related to birefringence within the subject [1].

\section{Materials and Methods}

Apparatus. The DOM apparatus consists of a transmissive liquid crystal display (LCD), microprocessor-based drive electronics, and software, all contained in a housing that can be secured to the light port of most microscopes by an included

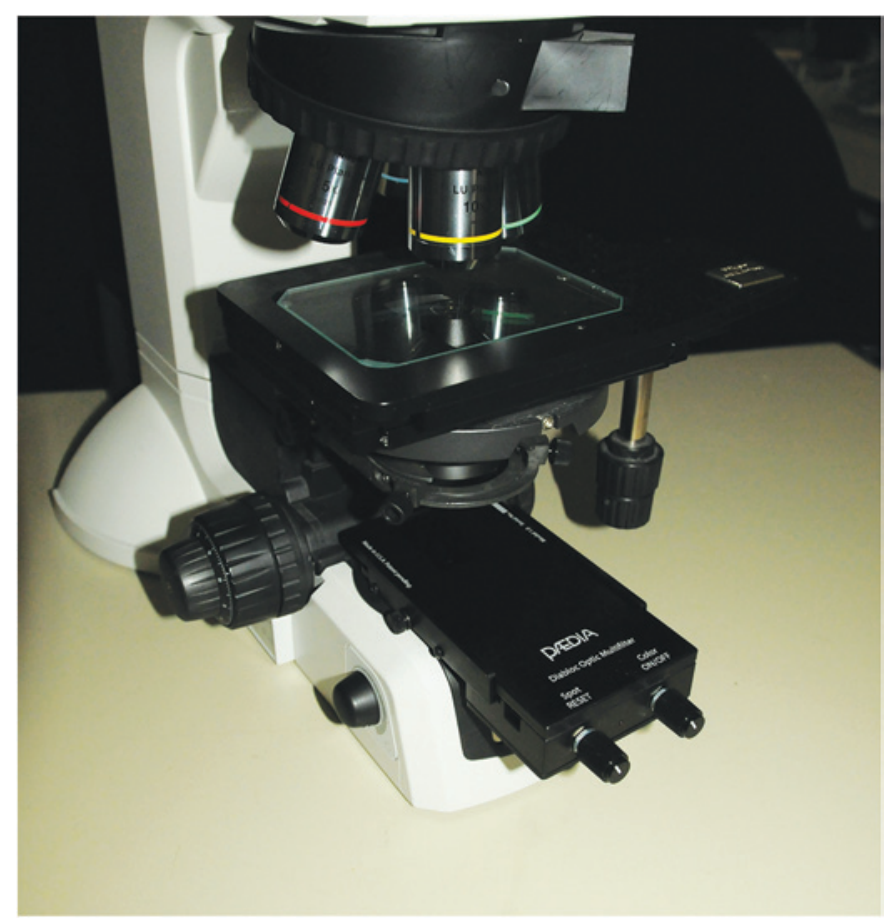

Figure 3: The DOM installed on a microscope. The Multifilter slides into a bracket that is secured to the base of the microscope.

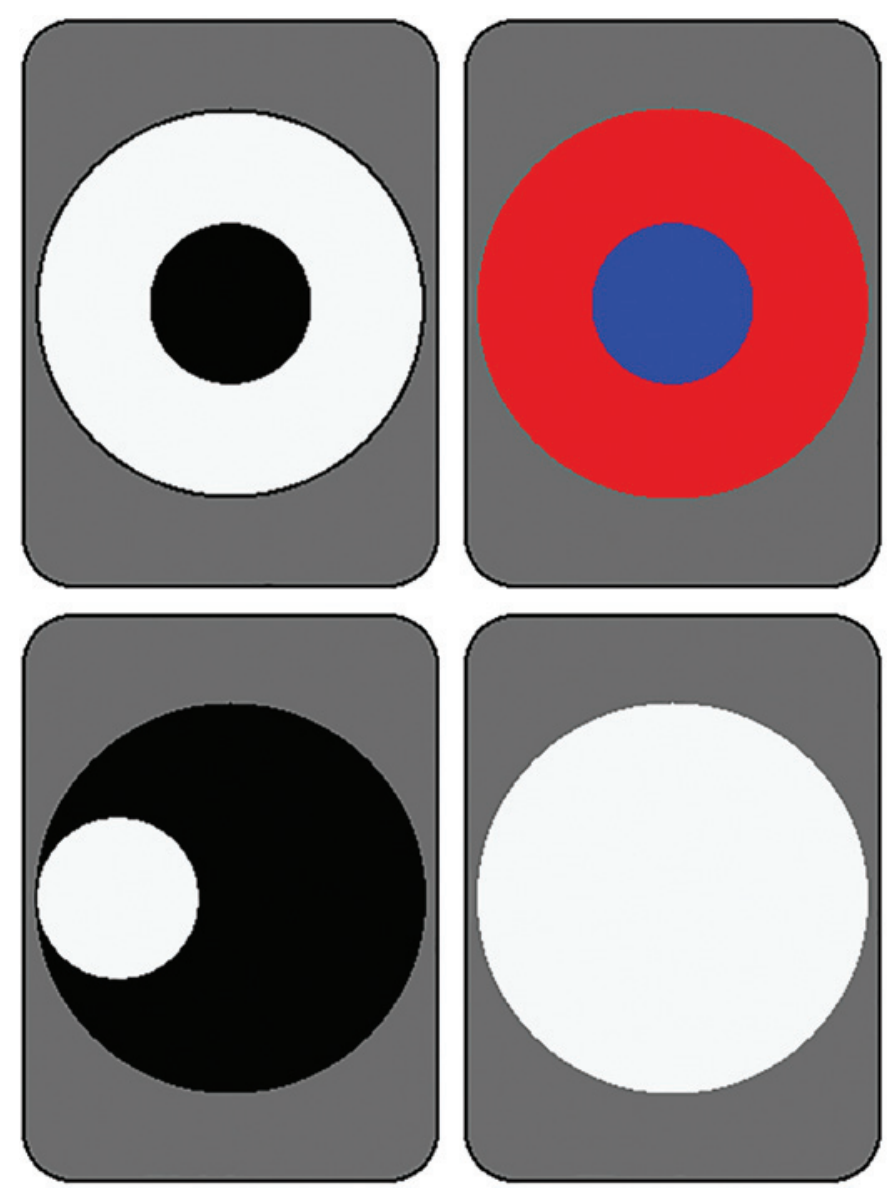

Figure 4: Selected filter patterns, clockwise from top left: darkfield, Rheinberg, monochrome or polarized, and oblique. Other patterns such as an annulus for use in phase contrast microscopy are easily generated. 

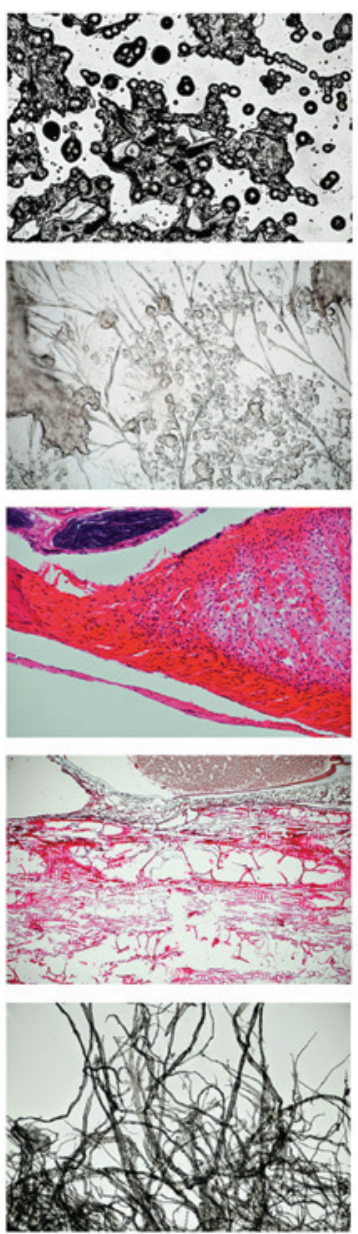

Bright field
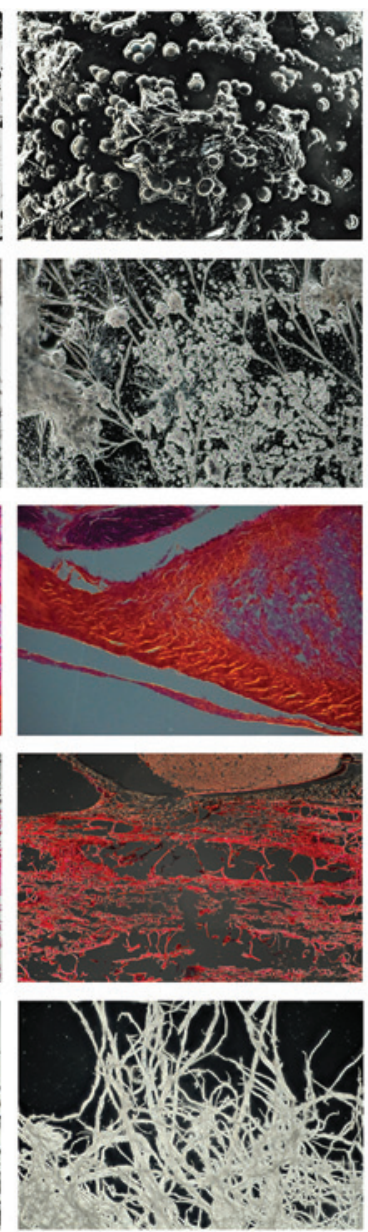

Dark field
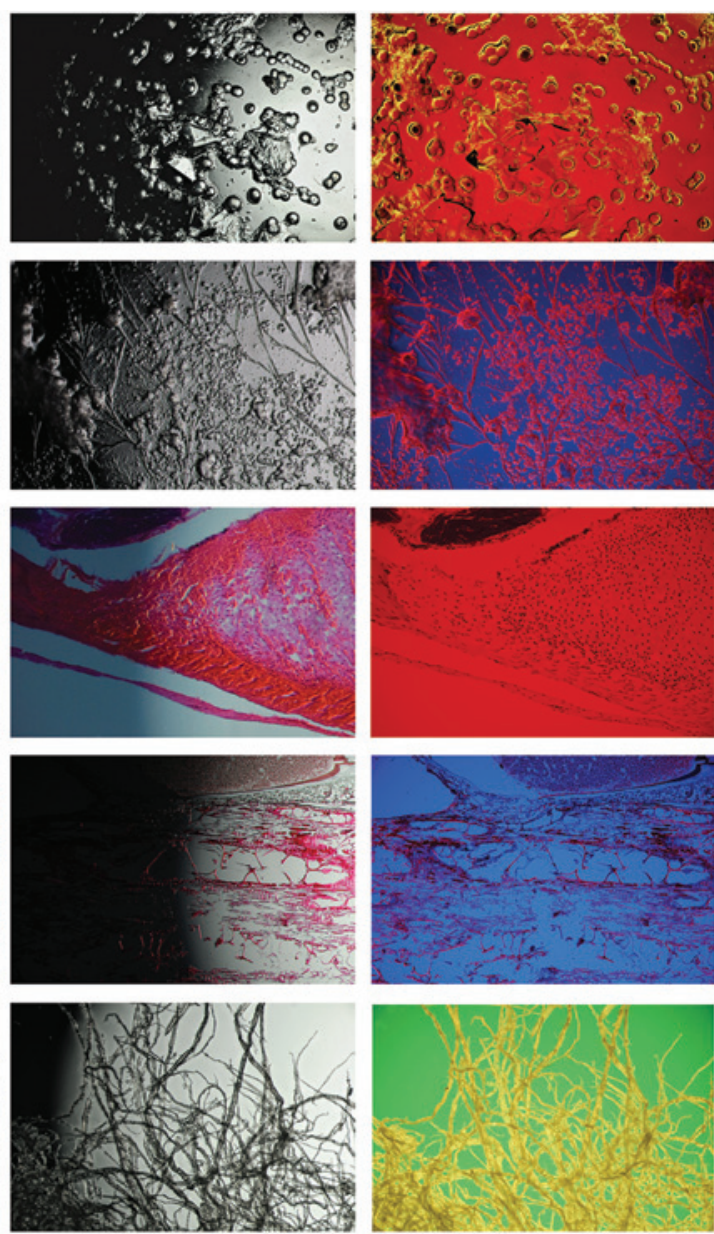

Oblique
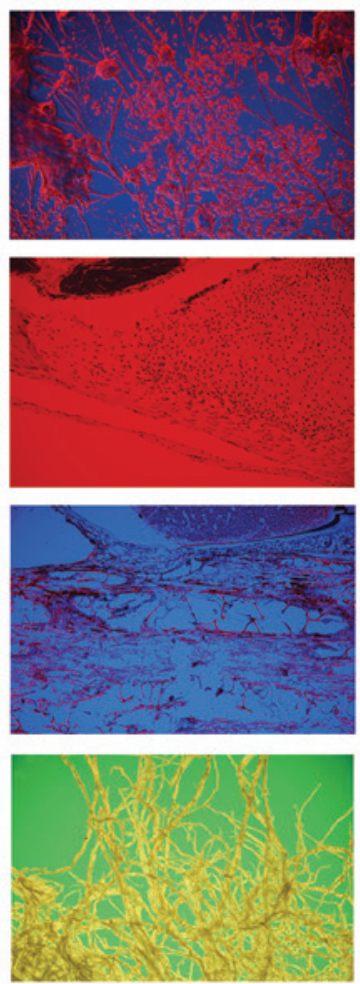

Rheinberg
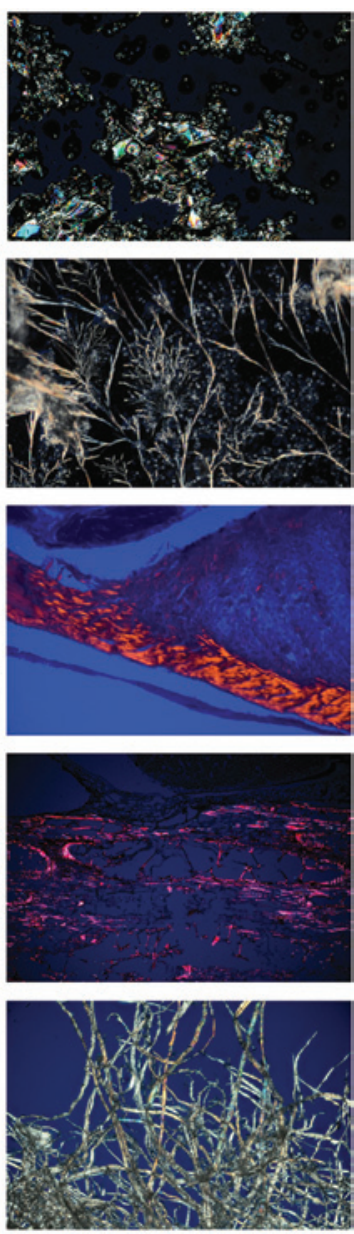

Polarized

Figure 5: Examples showing DOM contrast enhancement in chemical and biological samples. Top row: crystals formed from a solution of potassium, sodium, and calcium chloride salts; image width $=4.24 \mathrm{~mm}$. Second row: crystals from an aspirin tablet; image width $=4.24 \mathrm{~mm}$. Third row: mammalian muscle-tendon junction; image width $=1.06 \mathrm{~mm}$. Fourth row: Lycogala slime mold; image width $=2.12 \mathrm{~mm}$. Fifth row: torn edge of facial tissue; image width $=2.12 \mathrm{~mm}$.

mounting bracket. Operating power is derived from a wall supply, a USB connection, or a battery.

Figure 1 shows top, perspective, and side views of the DOM. Diabloc is an acronym for "diascopic light block." The $\mathrm{DOM}$ is $15 \times 7.5 \times 2.8 \mathrm{~cm}$ in length, width, and height. Figure 2 is a drawing showing placement of the DOM in the light path of a microscope. Figure 3 is a picture of the DOM in place on the light port of a microscope. Light from the microscope's lamp passes through the LCD and illuminates the microscope's condenser with computer-generated patterns.

Installation. Koehler illumination conditions [1] should be established in the microscope prior to installing the DOM. This ensures that the microscope's condenser is centered with respect to the microscope's light source and establishes the optimum condenser focus. Next, the microscope's field and condenser diaphragms are fully opened, and the DOM is installed and turned ON. A darkfield pattern appears in the DOM, and the dark center of the pattern is aligned with the microscope's optical axis by looking through the microscope's eyepiece. A mounting bracket supplied with the DOM permits easy removal and reinstallation of the DOM without the need for realignment. After these simple adjustments, the DOM is ready for use.
Operation. Figure 4 shows the light patterns generated in the LCD for the various illuminations described below. Clockwise from top left, they are darkfield, Rheinberg, monochrome or polarized, and oblique illuminations. The gray area indicates the outline of the LCD display. The large circle is the area occupied by a microscope's light source. The smaller circle is sizable and movable as a light block or light source, depending on the illumination method used. All functions of the DOM are controlled by two knobs located on the front of the unit. Colors are selected from combinations of varying intensities of red, green, and blue pixels on the display.

Darkfield. The basic reset condition of the DOM is a mid-sized dark spot in the center of a bright area on the LCD. This is the setup for darkfield illumination. The dark spot size is adjusted to just fill the field of view with the field and condenser irises fully open. A subject can now be examined in darkfield using objectives ranging from $5 \times$ to $20 \times$. Darkfield operation is fine-tuned with minor spot size and condenser focus adjustments.

Rheinberg. The setup for Rheinberg illumination is the same as for darkfield. Once the spot position and size are set, colors are introduced by turning the color knob on the DOM. 
Depressing the color knob toggles between inner and outer colors.

Brightfield and monochrome. For brightfield and monochrome illuminations, the field iris is fully open, and the condenser iris is set to a value equal to or slightly less than the numerical aperture of the objective lens in use. The DOM is reset, and the spot size knob on the DOM is turned until the central spot completely fills the bright area. For brightfield use, the color knob is turned one click so that the center spot is clear. For monochrome use, the color knob is turned until a desired filter color is reached. For example, a didymium filter is emulated by selecting a red-green-blue color combination with a minimal yellow component.

Polarized. To view a subject in polarized light, the field iris is fully open, and the condenser iris is set to a value equal to or slightly less than the numerical aperture of the objective lens in use. The DOM is set as for brightfield illumination. By virtue of its construction, light leaving an LCD is polarized. To view a subject in polarized light, an analyzer is added to the optical path above the microscope's objective lens.

Oblique. The setup for oblique illumination is similar to that for darkfield, except the roles of the dark spot and bright area on the LCD are reversed. Reversal is accomplished by turning the color knob. The field and condenser diaphragms on the microscope are fully opened. The position and size of the light spot on the LCD are adjusted by turning the spot control on the DOM.

Setup. The LCD chosen for use in the first Diabloc filter has linear polarizers on either side of a liquid crystal element. The polarizer nearest the light source has a matte finish. Light transmission through the LCD is about 6\%. In our work, a $50 \mathrm{~W}$ bulb in the microscope's lamp housing provides sufficient light intensity for all subjects we have seen. The white-toblack contrast ratio of the LCD is greater than 300:1 for a good darkfield outcome. An Abbe condenser was used in this work [1].

\section{Results}

Figure 5 shows a comparison of brightfield, darkfield, oblique, Rheinberg, and polarized illuminations on a variety of samples. The top row shows crystals formed from a solution of potassium, sodium, and calcium chloride salts. A $5 \times$ objective was used, and the image width is $4.24 \mathrm{~mm}$. The Rheinberg image was taken with a red spot and a yellow ring. The second row shows crystals obtained by dissolving and then crystallizing an aspirin tablet. The Rheinberg image was taken with a blue central spot and a red ring. The third row shows a mammalian muscle-tendon junction. A $20 \times$ objective was used, and the image width is $1.06 \mathrm{~mm}$. The Rheinberg image was taken with a red spot and a blue ring. The fourth row shows Lycogala species of plasmodial slime mold. A 10× objective was used, and the image width is $2.12 \mathrm{~mm}$. The Rheinberg image was taken with a blue central spot and a red ring. The fifth row shows a torn edge of commercial facial tissue. The Rheinberg image was taken with a green central spot and a yellow ring. The polarized image in the facial tissue example is a combination of darkfield and polarized illuminations. More information and more images are available at www.diabloc.com.

\section{Conclusion}

This article describes an all-in-one illumination filter for diascopic microscopy that provides brightfield, darkfield, oblique, Rheinberg, monochrome, and polarized illuminations in a single device. A digitally controlled transmissive LCD is interposed between a microscope's light source and condenser. Various patterns on the LCD are selectable and adjustable by turning two knobs on the front of the device. These patterns emulate the light-blocking and filtering components previously used with various contrast-enhancing illumination methods. Switching among illumination methods takes only a few seconds because there is no need to install a separate component for each method. Some methods can be combined for additional contrast and discrimination in an image.

\section{Acknowledgment}

Diabloc is a trademark of Paedia LLC. Paedia is a registered trademark and service mark of Paedia Corporation. Patent protection for the Diabloc illumination filter is pending.

\section{References}

[1] DB Murphy and MW Davidson, Fundamentals of Light Microscopy and Electronic Imaging, 2nd ed., Wiley-Blackwell, Hoboken, 2013.

[2] RO Wayne, Light and Video Microscopy, 2nd ed., Elsevier, London, 2014.

[3] http://micro.magnet.fsu.edu/primer/java/photomicrography/ didymium/index.html.

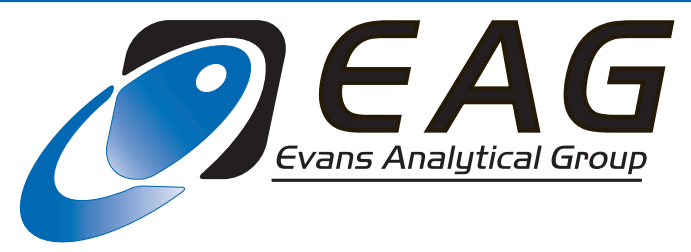

\section{Osiris with ChemiSTEM ${ }^{\mathrm{T}}$ Technology}

- Improved EDS mapping capability

- Increased sensitivity for light elements ( $3 \mathrm{x}$ more counts in O-K peak)

- Increased sensitivity for small concentrations Images and maps by D. Klenov, FEI

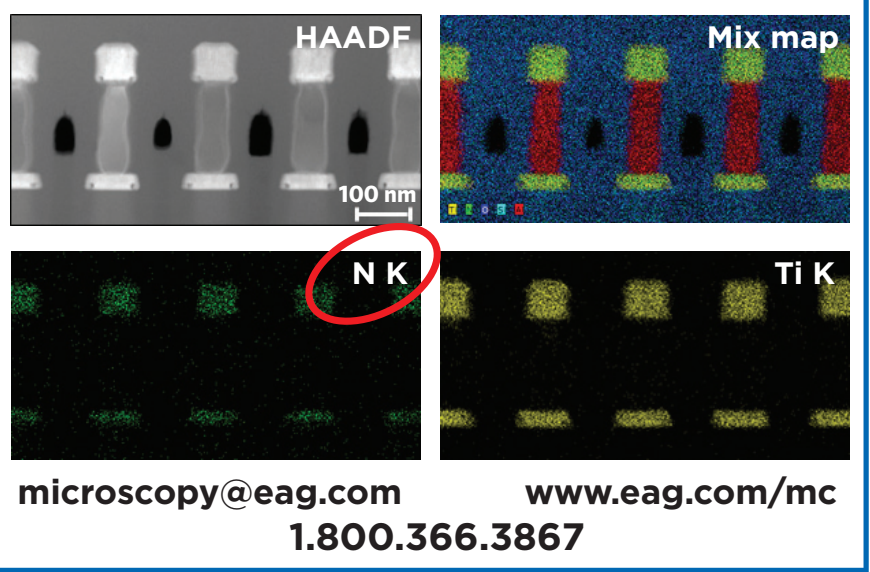

